\title{
The resurgence of Organic Photovoltaics
}

\author{
Ioannis lerides, Andrea Zampetti \& Franco Cacialli*
}

Department of Physics and Astronomy and London Centre for Nanotechnology, University College London, London WC1H OAH

E-mail: ioannis.ierides.17@ucl.ac.uk, a.zampetti@ucl.ac.uk, f.cacialli@ucl.ac.uk *

${ }^{*}$ Corresponding author

Keywords:

Organic photovoltaics, Non-fullerene acceptors, Ternary blends, Tandem solar cells, Organic semiconductors

\begin{abstract}
:
Several advances were reported in the field of organic photovoltaics in the last two years. Development and application of non-fullerene acceptors in particular has injected new life into the field. Exploitation of such materials in ternary blends and tandem solar cell structures has in fact enabled record high efficiencies $>15 \%$, thus paving the way towards commercialisation.
\end{abstract}

\section{Introduction:}

Organic semiconductors (OS) rapidly became a success story for light-emitting diodes and then displays, now found in a plethora of commercial applications with further current focus on extending their performance in the near infra-red [1,2]. On the other hand, organic photovoltaics (OPVs) have lagged behind due to their limited efficiencies. Recently, advances in OPV research led to the fabrication of record high $17.3 \%$ [3.•] efficient solar cells, thus demonstrating a potentially bright future for OPVs as well. The key competitive advantage of OPVs is their solutionprocessability which can offer low-cost fabrication of flexible devices over large areas [4]. They can also be semitransparent, and therefore penetrate into niche markets such as photovoltaic windows $[5,6]$. Indeed, OPVs have already shown excellent potential especially for wearable technologies and indoor energy generation $[7,8]$.

In the period 1980 to 2010 several milestones led to the increase of power conversion efficiencies (PCE) of OPVs from under $1 \%$ to approximately 10\% [9] (stated PCE values in this review refer to measurements taken under the typical AM 1.5G solar standard). First was the introduction of the bilayer structure [10]. Forming a heterojunction between electron donating (donor) and electron accepting (acceptor) OSs, allowed for efficient charge separation by providing a driving force to overcome the relatively high binding energy of excitons in OS $(\sim 0.5 \mathrm{eV})$. The advent of the bulk heterojunction (BHJ) concept that followed, allowed then formation of extended donor-acceptor interfaces and higher performance [11,12]. Fullerenes, and especially the soluble derivatives $\mathrm{PC}_{61} \mathrm{BM}$ and $\mathrm{PC}_{71} \mathrm{BM}$, quickly became the "elective" acceptors mainly due to their ability to form effective BHJs [13] (see Figure 1 for the chemical structures of all reported materials). In the years $2000-2010$ the efforts of the scientific community were directed to the careful optimisation of the morphology of active layers via additives and annealing, the development of interlayers to enhance charge transport to the electrodes (and extraction from these) and, most importantly, the synthesis of high-mobility and strongly absorbing donors [14]-[21]. Crucially, efforts in the last few years have included the development of non-fullerene acceptors (NFAs).

In this review we initially discuss advantages and limitations of single-junction OPVs based on fullerene acceptors, and then map out how developments in NFAs along with the effective use of the concepts of ternary blends and tandem cells allowed for a major enhancement in the performance of OPVs in the last two years.

\section{Fullerene acceptors:}

The dominance of fullerene utilisation in OPVs stems from their ability to form BHJ interpenetrating networks of good quality and their relatively high electron mobilities $\left(\sim 1 \times 10^{-2} \mathrm{~cm}^{2} / \mathrm{Vs}\right)[13]$. On the other hand, fullerenes suffer from relatively limited tunability of electron affinities [22], which in turn limits the number of potential donor materials that can be utilised and more importantly exhibit weak absorption in the visible and near infra-red regions, thus effectively limiting the maximum value of short circuit current densities $\left(J_{s c}\right)$. Furthermore, although there is evidence that the crystallinity of the fullerene phase influences OPV performance this lacks optimisation since the crystal phase depends on the solvent and is difficult to characterise $[23,24]$. These drawbacks restricted attainment of efficiencies above $15 \%$ for single junction OPVs based on donor:fullerene blends. Only recently has the crystal structure of solvent-free $\mathrm{PC}_{61} \mathrm{BM}$ single crystals been resolved and the morphological and optical properties of these crystals characterised [25]-[27•]. These studies can offer a pathway towards optimisation of fullerenes whose utilisation is likely to persist especially in ternary blends.

\section{Non- fullerene acceptors:}

The development of NFAs has become a topic of intense study as researchers are struggling to overcome fullerene challenges. In NFAs, energy levels and absorption spectra can be tuned more easily than in the case of fullerenes so as to optimise device function. The most widely studied classes of NFAs are perylene diimides (PDIs) and fusedring electron acceptors (FREAs) [28]. PDIs have been functionalised for use in BHJs in the positions shown in 
Figure 1d. Indeed the frontier energy levels (especially the lowest unoccupied molecular orbital (LUMO)) of NFAs are shown to be readily tuneable and to depend on the units attached to these positions. For example, Hartnett et al. [29] functionalised the ortho positions of the PDIs with the addition of hexyl groups (hexyl-PDI) and phenyl groups (phenyl-PDI). As expected, the different modifications led to different LUMO levels (3.83 eV and $4.01 \mathrm{eV}$ for hexyl-PDI and phenyl-PDI respectively) and energy gaps. Blending with PBTI3T resulted in a PCE of $3.6 \%$ for phenyl-PDI and $0.6 \%$ for hexyl-PDI. This significant discrepancy was attributed to the different morphologies of the blends. Transmission electron microscopy (TEM) revealed features $\sim 100 \mathrm{~nm}$ in length in PBTI3T:hexyl-PDI blends, thus suggesting poor mixing whereas PBTI3T:Phenyl-PDI blends showed homogeneous and well-mixed regions. These results exemplify a challenge in NFAs. Although the strong $\pi-\pi$ stacking in these systems leads to high mobilities $\left(\sim 0.2 \times 10^{-2} \mathrm{~cm}^{2} / \mathrm{Vs}\right)[30]$, formation of micrometre-sized crystallites is observed which significantly hinders performance in $\mathrm{BHJs}$.

The best performing NFAs are FREAs which consist of two strongly m-electron-withdrawing moieties linked by a planar $\pi$ bridge consisting of fused rings [31]. As expected, core size and the choice of end group significantly affect the optical and electrochemical properties of FREAs. To limit the formation of large domains of NFAs via m$\pi$ stacking, the fused rings are substituted with side chains that project above or below the plane. The added bonus is that these side chains can also be used to tailor solubility, mobility ,and active-layer morphology [30].

A very successful FREA is ITIC (Figure 1h) [32] developed by end capping IT with a so called INCN moiety. INCN features one carbonyl and two cyano groups that downshift LUMO levels thanks to their electron-withdrawing character. The four (rigid) hexylphenyl groups helped to form PTB7-Th:ITIC blends that had nearly uniform crystalline grains $(\sim 10 \mathrm{~nm})$, resulting in PCEs $\sim 6.8 \%$. When Haijun et al. [33••] blended ITIC with a donor with complementary absorption spectrum (J71 - Figure 1p), they increased the PCE to $11.4 \%$, and the $J_{s c}$ from 14.3 to $17.32 \mathrm{~mA} / \mathrm{cm}^{2}$. Incident photon to current efficiency measurements of devices based on $\mathrm{J} 71: \mathrm{ITIC}$ blends showed broad and efficient photo-response from 300 to $790 \mathrm{~nm}$, indicating high photo-conversion efficiency for the absorptions of both $\mathrm{J} 71$ and ITIC. Transient absorption measurements also confirmed that hole transfer takes place at pump wavelengths where only ITIC is excited. These results show that charge transfer from excited states formed both in the donor and the acceptor can occur effectively in NFA blends, leading to higher $J_{s c}$ with respect to fullerene-based blends [33••, 34]. These findings are particularly interesting since they provide design rules for NFA OPV systems. Further PCE enhancements were achieved by Zhao et al. [35॰] by performing fluorination of ITIC as shown in Figure 1i (IT-4F). By exploiting the enhanced absorption and reduced energy gap of IT-4F, they obtained a $13.1 \%$ efficiency for single junction OPVs using PBDB-T-SF as donor. Another noteworthy example of a NFA is COi8DFIC [36•]. This low bandgap NFA (1.26 eV) exhibited an outstanding $J_{s c}$ of $26.12 \mathrm{~mA} / \mathrm{cm}^{2}$ with a $12.16 \%$ PCE in PTB7-Th:COi8DFIC blends. This impressive result was attributed to the complementary absorption and well-balanced mobilities of PTB7-Th and COi8DFIC $\left(\sim 10^{-4} \mathrm{~cm}^{2} / \mathrm{Vs}\right)$.

Overall, a variety of advances in NFAs since 2015 increased OPV PCEs from $6 \%$ to $13 \%$. Electron affinities can be effectively tailored over a very wide range of structures and the knowledge and techniques that have been previously applied to donors can be exploited [37]. Large, medium, and small energy gap NFAs are now available and can accommodate essentially all types of donors to achieve complementary spectral features. This allows high $J_{S c}$ and PCEs to be attained. However, it is not yet clear that interpenetration of donor and acceptor moieties in NFA BHJs is as good as in the case of fullerene BHJs. Effectively tackling this challenge to ensure the formation of effective BHJs is very likely to lead to even greater improvements in OPVs incorporating NFAs.

\section{Ternary blends and tandem solar cells}

The concepts of ternary blends and tandem/multiple solar cells are now widely applied to photovoltaics to counter thermalisation losses. These concepts are particularly useful for OPVs as well, as the coverage of the solar irradiation spectrum is not optimal in single junction donor:acceptor BHJs due to the relatively narrow optical absorption of OS when compared to silicon for example.

Lu et al. [38•-0] were among the first to combine fullerenes and NFAs in ternary blends [39,40]. This innovative approach partially eliminates the deficiencies of these two types of acceptors by capitalising on their complementary advantages. In their study [38••], PPBDTBT was used as a donor, with ITIC and PC ${ }_{71}$ BM used as acceptors. To enable comparison, devices of binary blends containing only a single acceptor were fabricated as well. The external quantum efficiency spectra of the ternary device had a similar profile to ITIC-only devices with enhanced values in every region of the spectrum. This indicated that whilst most absorption was resulting from ITIC and the donor, photogenerated excitons were dissociated and collected more effectively in the ternary blend. Furthermore, the ternary device was 35\% and $43 \%$ more efficient than the binary devices of NFAs and fullerenes respectively. Increasing the weight percentage of fullerene content in the ternary blend increased the fill factor as well. A maximum $J_{s c}$ was reached at $40 \% \mathrm{PC}_{71} \mathrm{BM}$ weight concentration, with a corresponding $\mathrm{PCE}=10.4 \%$. Interestingly, these results clearly demonstrated that the NFA enhances light absorption in these systems, while the fullerene improves the morphology and charge-carrier transport [38••]. The synergistic use of non-fullerene and fullerene acceptors in ternary blends was also explored by Zhao et al. [41] and Xiao et al. [42•] to boost the PCE. They achieved $12.2 \%$ and $14 \%$ PCEs respectively. Xiao et al. used a blend of PTB7-Th, COi8DFIC and PC ${ }_{71} B M$ thus obtaining an impressive $28.20 \mathrm{~mA} / \mathrm{cm}^{2} J_{s c}$ and a $71 \%$ fill factor. 
Ternary blends have also been used effectively in tandem structures $[3 \bullet, 43]$ which are particularly useful for OPVs, because OS generally have low mobilities and require active layers of limited thickness compared to inorganic semiconductors [44].

The most successful tandem OPVs to date have been demonstrated by Meng et al. [3••]. In their study, material selection was guided by a semi-empirical analysis from which several desired parameters for an optimised system were extracted. For example, the optimal absorption onsets of the rear and front sub-cells were calculated to be $1050-1150 \mathrm{~nm}$ and $720 \mathrm{~nm}$ respectively. For their experimental investigations they chose a rear cell constructed by the ternary blend: PTB7-Th, COi8DFIC, PC ${ }_{71}$ BM and a PBDB-T: F-M blend front cell to match the criteria. They also employed computational simulations to determine the optimised active layer thickness. The ensuing strategy enabled fabrication of solution-processed devices with PCEs up to $17.3 \%$, currently the state-of-the-art. This outstanding result stresses the importance of using computational models to guide experimental attempts and more importantly revives interest in the exciting field of OPVs.

\section{The path to commercialisation:}

PCEs $>15 \%$ in fully solution-processed devices, prove OPVs promise as a viable technology for commercialisation. Currently, the biggest obstacle is lack of long-term stability. Although most recent devices containing NFAs report improved stabilities, a deeper understanding is required to elucidate stability to oxygen, moisture, heat, irradiation and mechanical stress $[45,46]$. For OPVs to fulfil their potential they must also compete with currently growing and established photovoltaic technologies. As lead-free devices, OPVs have an inherent competitive advantage over lead-halide perovskites [47] despite affording lower PCEs. Competition from silicon and copper indium gallium selenide (CIGS) solar cells is possibly even stronger. Although OPVs can be solution-processed, thus offering potentially lower fabrication costs, these established solar technologies already benefit from economies of scale (especially silicon). However, OPVs tend to perform better under indoor illumination conditions [48] and can be flexible. The growing interest in applications where these properties are required can offer a window of opportunities for commercialisation of OPVs. The better integration of computational simulations and experimental techniques seems to be a very fruitful avenue to pursue and will likely play a major role in the future development of OPVs to mitigate challenges in both non-fullerene and fullerene acceptor devices.[49]

\section{Conclusion:}

In the last two years we have witnessed significant developments in the field of organic photovoltaics. Better understanding and application of design rules for non-fullerene acceptors has led to an increase in the power conversion efficiency of single junction organic photovoltaics that employ them by a factor of 2 . Great strides have also been made in ternary blends by the innovative approach of effectively combining fullerene and non-fullerene acceptors. The $15 \%$ power conversion efficiency benchmark has been surpassed in tandem structures by bringing together guidance from computational models and these ternary blends containing the state-of-the-art nonfullerene acceptors. With the revived interest in organic photovoltaics that these advances bring, we can expect attainment of even higher efficiencies as well as the development of strategies to mitigate issues with stability of organic photovoltaics in the near future.

\section{Acknowledgements:}

II was supported by the EPSRC Centre for Doctoral Training in Advanced Characterisation of Materials (grant number EP/L015277/1). FC is a Royal Society Wolfson Merit Award holder.

\section{Declaration of interest: None}

\section{References and recommended reading:}

Papers have been highlighted as:

- of special interest

•• of outstanding interest

[1] A. Minotto, P. Murto, Z. Genene, A. Zampetti, G. Carnicella, W. Mammo, M. R. Andersson, E. Wang \& F. Cacialli, "Efficient Near-Infrared Electroluminescence at $840 \mathrm{~nm}$ with 'Metal-Free' Small-Molecule: Polymer Blends", Adv. Mater., 30, 1706584, (2018).

[2] A. Zampetti, A. Minotto, B. M. Squeo, V. G. Gregoriou, S. Allard, U. Scherf, C. L. Chochos \& F. Cacialli, "Highly Efficient Solid-State Near-infrared Organic Light-Emitting Diodes incorporating A-D-A Dyes based on $\alpha, \beta-$ unsubstituted 'BODIPY' Moieties”, Sci. Rep., 7, 1611, (2017).

[3••] L. Meng, Y. Zhang, X. Wan, C. Li, X. Zhang, Y. Wang, X. Ke, Z. Xiao, L. Ding, R. Xia, H.L. Yip, Y. Cao \& Y. Chen, "Organic and solution-processed tandem solar cells with $17.3 \%$ efficiency", Science, 10,1126, (2018).

This paper outlines the development of the most efficient OPV to date. It demonstrates how bringing computational models and state-of-the-art materials can be an effective method to maximise performance.

[4] L. Mao, J. Tong, S. Xiong, F. Jiang, F. Qin, W. Meng, B. Luo, Y. Liu, Z. Li, Y. Jiang, C. Fuentes-Hernandez, B. Kippelenb \& Y. Zhou, "Flexible large-area organic tandem solar cells with high defect tolerance and device yield", J. Mater. Chem., 5, 7, (2017). 
[5] Q. Tai \& F. Yan, "Emerging Semitransparent Solar Cells: Materials and Device Design", Adv. Mater., 29, 34, (2017).

[6] Y. Li, G. Xu, C. Cui \& Y. Li, "Flexible and Semitransparent Organic Solar Cells", Adv. Energy Mater., 8, 7, (2018). [7] H. Yin, J. K. W. Ho, S. H. Cheung, R. J. Yan, K. L. Chiu, X. Hao \& S. K. So, "Designing a ternary photovoltaic cell for indoor light harvesting with a power conversion efficiency exceeding 20\%", J. Mater. Chem. A, 6, 18, (2018). [8] H. K. H. Lee, J. Wu, J. Barbe, S. M. Jain, S. Wood, E. M. Speller, Z. Li, F. A. Castro, J. R. Durrant \& W. C. Tsoi, "Organic photovoltaic cells-promising indoor light harvesters for self-sustainable electronics", J. Mater. Chem. A, 6, 14, (2018).

[9] J. Yu, Y. Zheng \& J. Huang, "Towards high performance organic photovoltaic cells: A review of recent development in organic photovoltaics", Polymers, 6, 9, (2014).

[10] C. W. Tang, "Two-layer organic photovoltaic cell," Appl. Phys. Lett., 48, 2, (1986).

[11] G. Yu, J. Gao, J. C. Hummelen, F. Wudl \& A. J. Heeger, "Polymer Photovoltaic Cells: Enhanced Efficiencies via a Network of Internal Donor-Acceptor Heterojunctions" Science, 270, 5243, (1995).

[12] J. J. M. Halls, C. A. Walsh, N. C. Greenham, E. A. Marseglia, R. H. Friend, S. C. Moratti \& A. B. Holmes, "Efficient photodiodes from interpenetrating polymer networks" Nature, 376, (1995).

[13] C. B. Nielsen, S. Holliday, H. Y. Chen, S. J. Cryer \& I. McCulloch, "Non-Fullerene Electron Acceptors for Use in Organic Solar Cells", Acc. Chem. Res., 48, 11, (2015).

[14] Y. Liang, Z. Xu, J. Xia, S. Tsai, Y. Wu, G. Li, C. Ray \& L. Yu, "For the bright future-bulk heterojunction polymer solar cells with power conversion efficiency of 7.4\%", Adv. Mater., 22, 20, (2010).

[15] H. Y. Chen, J. Hou, S. Zhang, Y. Liang, G. Yang, Y. Yang, L. Yu, Y. Wu \& G. Li, "Polymer solar cells with enhanced open-circuit voltage and efficiency", Nat. Photonics, 3, 11, (2009).

[16] Y. Liang, Y. Wu, D. Feng, S. Tsai, H.J. Son, G. Li \& L. Yu, "Development of new semiconducting polymers for high performance solar cells", J. Am. Chem. Soc., 131, 1, (2009).

[17] G. Winroth, D. Podobinski \& F. Cacialli, "Dopant optimization for triplet harvesting in polymer photovoltaics", J. Appl. Phys., 110, 12, (2011).

[18] P. Li, O. Fenwick, S. Yilmaz, D. Breusov, D. J. Caruana, S. Allard, U. Scherf \& F. Cacialli "Dual functions of a novel low-gap polymer for near infra-red photovoltaics and light-emitting diodes", Chem. Commun., 47, 31, (2011). [19] G. Paternó, F. Cacialli \& V. García-Sakai, "Structural and dynamical characterization of P3HT/PCBM blends", Chem. Phys., 427, (2013).

[20] N. Seidler, G. M. Lazzerini, G. L. Destri, G. Marletta \& F. Cacialli, "Enhanced crystallinity and film retention of P3HT thin- films for efficient organic solar cells by use of preformed nano fibers in solution", 1, 7748, (2013).

[21] R. Riehn, R. Stevenson, D. Richards, D. Kang, M. Blamire, A. Downes \& F. Cacialli, "Local probing of photocurrent and photoluminescence in a phase-separated conjugated-polymer blend by means of near-field excitation", Adv. Funct. Mater., 16, 4, (2006).

[22] R. Ganesamoorthy, G. Sathiyan \& P. Sakthivel, "Review: Fullerene based acceptors for efficient bulk heterojunction organic solar cell applications," Sol. Energy Mater. Sol. Cells, 161, (2017).

[23] G. M. Paternò, M. W. A. Skoda, R. Dalgliesh, F. Cacialli \& V. García Sakai, "Tuning fullerene intercalation in a Poly (thiophene) derivative by controlling the polymer degree of self-organisation", Sci. Rep., 6, (2016).

[24] G. M. Paternò, J. R. Stewart, A. Wildes, F. Cacialli \& V. G. Sakai, "Neutron polarisation analysis of Polymer:Fullerene blends for organic photovoltaics", Polymer, 105, (2016).

[25] G. Tregnago, M. Wykes, G. M. Paternò, D. Beljonne \& F. Cacialli, "Low-temperature photoluminescence spectroscopy of solvent-free PCBM single-crystals", J. Phys. Chem. C, 119, 21, (2015).

[26] G. M. Paternò, A. J. Warren, J. Spencer, G. Evans, V. G. Sakai, J. Blumberger \& F. Cacialli, "Micro-focused X-ray diffraction characterization of high- quality [6,6]-phenyl-C61-butyric acid methyl ester single crystals without solvent impurities", 1, 36, (2013).

[27•] G. M. Lazzerini, G. M. Paternò, G. Tregnago, N. Treat, N. Stingelin, A. Yacoot \& F. Cacialli "Traceable atomic force microscopy of high-quality solvent-free crystals of [6,6]-phenyl-C61-butyric acid methyl ester", Appl. Phys. Lett., 108, 5, (2016).

This paper, shows the significance of solvent evaporation to the finalised crystal phase formation of $P C_{61} B M$. Optimisation and control of crystal phase is important as it affects the PCE of OPVs.

[28] P. Cheng, G. Li, X. Zhan \& Y. Yang, "Next-generation organic photovoltaics based on non-fullerene acceptors", Nat. Photonics, 12, 3, (2018).

[29] P. E. Hartnett, A. Timalsina, H. S. S. R. Matte, N. Zhou, X. Guo, W. Zhao, A. Facchetti, R. P. H. Chang, M. C. Hersam, M. R. Wasielewski \& T. J. Marks, "Slip-stacked perylenediimides as an alternative strategy for high efficiency nonfullerene acceptors in organic photovoltaics", J. Am. Chem. Soc., 136, 46, (2014).

[30] C. Yan, S. Barlow, Z. Wang, H. Yan, A. K. Y. Jen, S. R. Marder \& X. Zhan, "Non-fullerene acceptors for organic solar cells", Nat. Rev. Mater., 3, (2018).

[31] Y. Lin \& X. Zhan, "Designing efficient non-fullerene acceptors by tailoring extended fused-rings with electrondeficient groups", Adv. Energy Mater., 5, 20, (2015).

[32] Y. Lin, J. Wang, Z. Zhang, H. Bai, Y. Li, D. Zhu \& X.i Zhan, "An electron acceptor challenging fullerenes for efficient polymer solar cells", Adv. Mater., 27, 7, (2015).

[33.•] H. Bin, L. Gao, Z. Zhang, Y. Yang, Y. Zhang, C. Zhang, S. Chen, L. Xue, C. Yang, M. Xiao \& Y. Li, "11.4\% Efficiency non-fullerene polymer solar cells with trialkylsilyl substituted 2D-conjugated polymer as donor", Nat. Commun., 7, (2016).

An excellent demonstration of the advantage of NFAs. Using transient absorption spectroscopy the authors show that both the donor and acceptor contribute to the Jsc. 
[34] J. Liu, S. Chen, D. Qian, B. Gautam, G. Yang, J. Zhao, J. Bergqvist, F. Zhang, W. Ma, H. Ade, O. Inganäs, K. Gundogdu, F. Gao \& H. Yan, "Fast charge separation in a non-fullerene organic solar cell with a small driving force", Nat. Energy, 1, 7, (2016).

[35•] W. Zhao, S. Li, H. Yao, S. Zhang, Y. Zhang, B. Yang \& J. Hou, "Molecular Optimization Enables over 13\% Efficiency in Organic Solar Cells", J. Am. Chem. Soc., 139, 21, (2017).

In this paper, fluorination which is a widely applied technique for donors, is shown to work effectively on NFAs as well, decreasing the bandgap and enhancing absorption resulting to a very efficient single junction OPV.

[36•] Z. Xiao, X. Jia, D. Li, S. Wang, X. Geng, F. Liu, J. Chen, S. Yang, T. P. Russell \& L. Ding, "26 mAcm-2 Jsc from organic solar cells with a low-bandgap nonfullerene acceptor", Sci. Bull., 62, 22, (2017).

The very promising NFA COi8DFIC is presented for the first time, showing outstanding performance.

[37] Q. Fan, W. Su, Y. Wang, B. Guo, Y. Jiang, X. Guo, F. Liu, T. P. Russell, M. Zhang \& Y. Li, "Synergistic effect of fluorination on both donor and acceptor materials for high performance non-fullerene polymer solar cells with $13.5 \%$ efficiency", Sci. China Chem., 61, 5, (2018).

[38••] H. Lu, J. Zhang, J. Chen, Q. Liu, X. Gong, S. Feng, X. Xu, W. Ma \& Z. Bo, "Ternary-Blend Polymer Solar Cells Combining Fullerene and Nonfullerene Acceptors to Synergistically Boost the Photovoltaic Performance", Adv. Mater., 28, 43, (2016).

The innovative approach of combining non-fullerene and fullerene acceptors in ternary blends is presented for the first time. Mixing of the two acceptors is harmless to performance and the overall structure benefits from the advantages of each acceptor whilst the disadvantages are diminished.

[39] Y. Chen, P. Ye, Z. Zhu, X. Wang, L. Yang, X. Xu, X. Wu, T. Dong, H. Zhang, J. Hou, F. Liu \& H. Huang, "Achieving High-Performance Ternary Organic Solar Cells through Tuning Acceptor Alloy", Adv. Mater., 29, 6, (2017).

[40] W. Xu \& F. Gao, "The progress and prospects of non-fullerene acceptors in ternary blend organic solar cells", Mater. Horizons, 5, 2, (2018).

[41] W. Zhao, S. Li, S. Zhang, X. Liu \& J. Hou, "Ternary Polymer Solar Cells based on Two Acceptors and One Donor for Achieving 12.2\% Efficiency", Adv. Mater., 29, 2, (2017).

[42•] Z. Xiao, X. Jia \& L. Ding, "Ternary organic solar cells offer $14 \%$ power conversion efficiency", Sci. Bull., 62, 23, (2017).

State of the art NFA COi8DFIC is shown to work effectively in ternary blends.

[43] Y. Li , J. Lin, X. Liu, Y. Qu, F. Wu, F. Liu, Z. Jiang \& S. R. Forrest, "Near-Infrared Ternary Tandem Solar Cells", Adv. Mater., 1804416, (2018).

[44] T. Ameri, G. Dennler, C. Lungenschmied \& C. J. Brabec, "Organic tandem solar cells: A review", Energy Environ. Sci., 2, 4, (2009).

[45] W. R. Mateker \& M. D. McGehee, "Progress in Understanding Degradation Mechanisms and Improving Stability in Organic Photovoltaics", Adv. Mater., 29, 10, (2017).

[46] Y. Zhang, I. D. W. Samuel, T. Wang \& D. G. Lidzey, "Current Status of Outdoor Lifetime Testing of Organic Photovoltaics", Adv. Sci., 5, 8, (2018).

[47] Q. Lin, A. Armin, P. L. Burn \& P. Meredith, "Organohalide Perovskites for Solar Energy Conversion", Acc. Chem. Res., 49, (2016).

[48] C. L. Cutting, M. Bag \& D. Venkataraman, "Indoor light recycling: A new home for organic photovoltaics", J. Mater. Chem. C, 4, 43, (2016).

[49] A. Zampetti, A. H. Fallahpour, M. Dianetti, L. Salamandra, F. Santoni, A. Gagliardi, M. A. der Maur, F. Brunetti, A. Reale, T. M. Brown \& A. Di Carlo "Influence of the interface material layers and semiconductor energetic disorder on the open circuit voltage in polymer solar cells", J. Polym. Sci., 53, 10, (2015). 
To appear in "Current Opinion in Green and Sustainable Chemistry" June 2019, Special Issue on Novel materials for energy production and storage edited by Yamashita K., Fabris S., Palummo M. and Giacomo G.

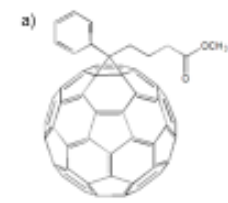

b)
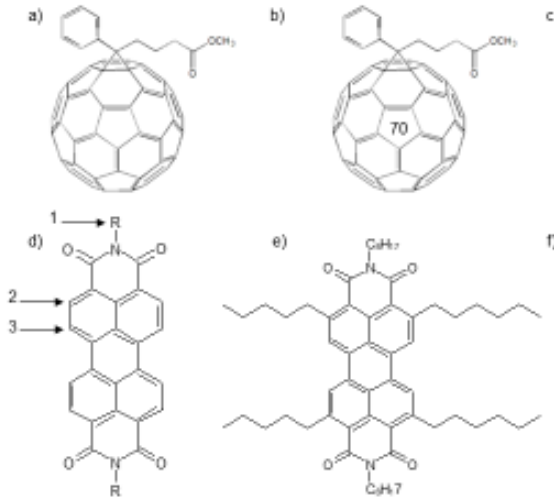

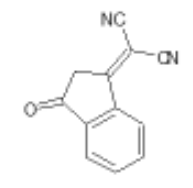

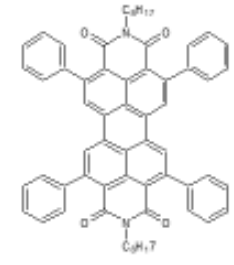

g)
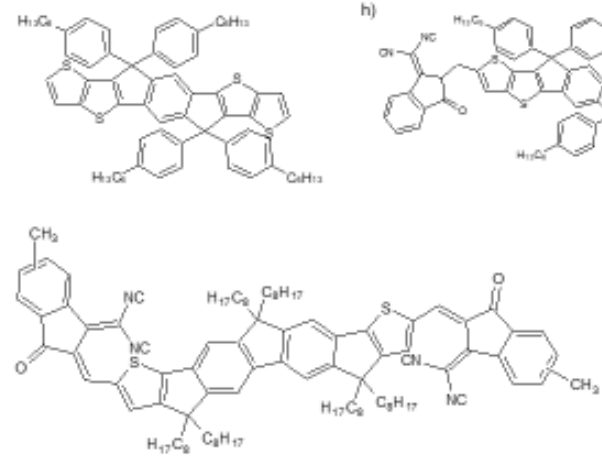
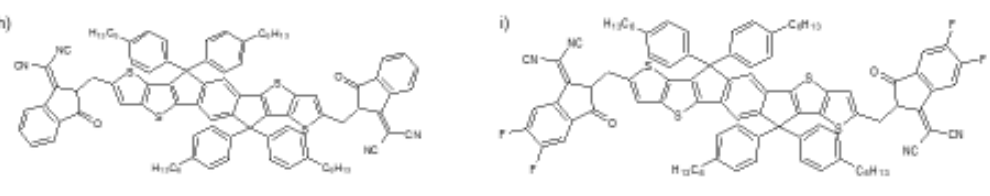

k)

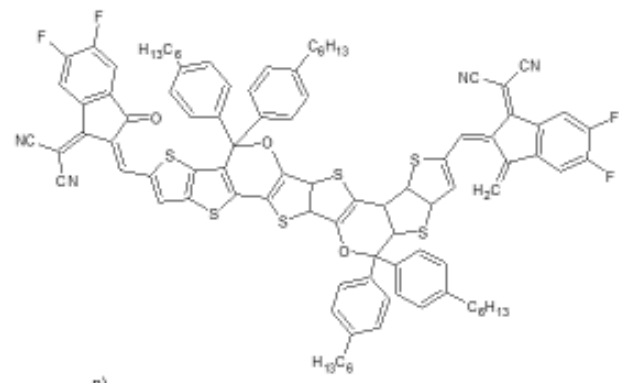

m)

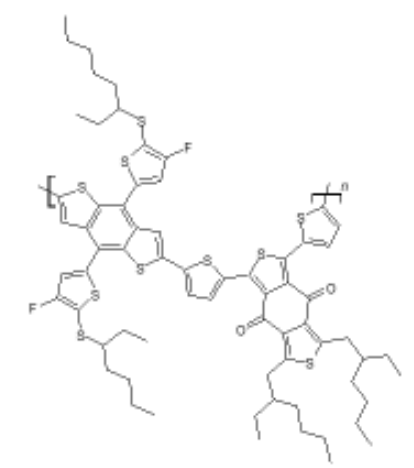

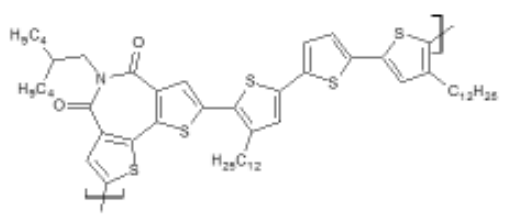

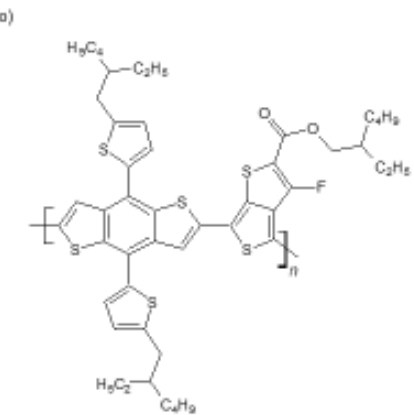

a)

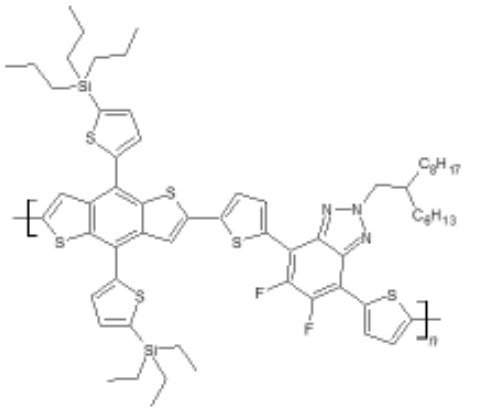

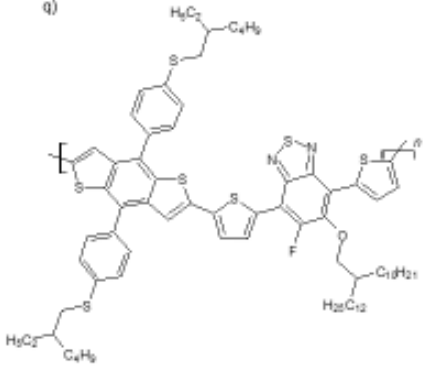

Figure 1: Reported chemical structures: a) $P C_{61} B M$, b) $\left.P C_{71} B M, c\right) I N C N$ d) $P D I$ (1.imide functionalisation, 2.ortho functionalisation 3. bay functionalisation), e) Hexyl-PDI, f) Phenyl-PDI, g) IT, h) ITIC, i) IT-4F, j) F-M, k) COi8DFIC, l) PBDB-T, m) PBDB-T-SF, n) PBTI3T, o) PTB7-TH, p) J71, q) PPBDTBT. 